\title{
Resultado anormal de la citología vaginal durante la gestación: 7 años de experiencia
}

\author{
Dr. Antonio González Mazuelo*; Dr. Néstor Villota Cadena*; Dra. Marisol Agudelo**; Dra. Guadalupe Posada**
}

Recibido: Julio 21 / 1999

Revisado: Agosto 2 / 1999

Aceptado: Junio $9 / 2000$

\section{RESUMEN}

Para correlacionar y destacar la importancia de la colposcopia en pacientes gestantes con citología anormal, se hizo un estudio retrospectivo en la ESE Metrosalud-Castilla de Medellín, de Mayo de 1991 a Junio de 1998, se tabularon datos de 127 pacientes, el promedio de edad fue de 24 años, la citología más frecuente fue de LIE de bajo grado (63\%). Se observó que en nuestro medio no existe una buena correlación citología-biopsia, pues se diagnosticaron 2 carcinomas en citologías de bajo grado y 5 carcinomas en citologías de alto grado.

Se sugiere por tanto como protocolo de manejo para pacientes embarazadas con citología anormal: colposcopia, toma de biopsia ante hallazgos dudosos, cambios mayores o invasión, conización diagnóstica para sospecha de microinvasión, tratamiento diferido al postparto para lesiones NIC y para el cáncer, tratamiento inmediato según normas del Instituto Nacional de Cancerología.

PALABRAS CLAVES: Citología y embarazo, colposcopia y embarazo.

\section{SUMMARY}

To correlate and to highlight the importance of the colposcopia in pregnant patient, with abnormal cytology; a retrospective study was made in the Metrosalud-Castilla of Medellín, of May of 1991 to June of 1998. 127 patients data were tabulated, the age average was of 24 biopsy doesn't exist, because 2 carcinomas were diagnosed in cytologies of low degree and 5 carcinomas in cytologies of high degree.

It is suggested therefore as handling protocol for pregnant patients with abnormal cytology: colposcopy, taking of biopsy before doubtful discoveries, bigger changes or evidence of invasive cancer; diagnostic conization for microinvasión suspicion. Treatment differed to the postparto for lesions CIN; patients with cancer should be treated according to norms of the National Institute of Cancer.

KEY WORDS: pregnancy, cytology, colposcopy.

\section{Introducción}

El problema de la citología anormal durante el embarazo se ha vuelto muy frecuente en nuestro medio y complica hasta el $5 \%$ de las pacientes que se hacen citología durante la gestación. Los amplios criterios citológicos para la sospecha de lesiones de bajo grado ha hecho que, en el momento actual, con la nueva terminología del grupo de Bethesda, sea aun más elevada.

Alrededor de un $10 \%$ de las mujeres con citología sugestiva de cambios de bajo grado tienen NIC II o NIC III y aunque el cáncer cervical es raro, se puede presentar.

Parece ser rara la asociación del cáncer cervico-uterino a la gestación. Sólo el $1 \%$ de las pacientes a quienes se diagnostica un cáncer cervical están al mismo tiempo embarazadas, y que una paciente gestante tenga concomitantemente un carcinoma sólo se presenta entre 1 y 1,5 de cada mil embarazos (1).

Sin embargo, la tendencia puede estar cambiando. La incidencia, al parecer creciente, de carcinoma cervicouterino en mujeres jóvenes y el hábito moderno de las mujeres a retrasar la procreación pudieran estar alterando las estadísticas de cáncer cervical y gestación. La edad modal del diagnóstico de las lesiones NIC II y NIC III disminuyó de 35 años en la década de los 60 a 25 años en $\operatorname{los} 90$ (1).

Por lo anterior, debe hacerse una evaluación juiciosa del programa de tamizaje citológico en toda gestante durante el control prenatal. Si éste no se ha iniciado o está incompleto, la obtención de un frotis cervico-uterino debe ser una norma de los cuidados prenatales, ya que un porcentaje importante de mujeres que se embarazan están en riesgo de neoplasia intraepitelial.

Como la mujer con cáncer cervico-uterino avanzado no se embaraza, el aspecto más importante del estudio citológico durante la gestación es excluir la posibilidad 
de un cáncer temprano, que usualmente suele ser una lesión pequeña y localizada, casi siempre oculta, y modificada por los cambios fisiológicos que son drásticos en el cérvix de las gestantes.

El manejo moderno del frotis de Papanicolau anormal durante la gestación, exige un estudio colposcópico sistemático por personal experimentado. Los cambios usuales de la gestación requieren destreza tanto para la realización como en la interpretación del examen.

Desde el punto de vista histórico, la primera etapa del manejo de la citología anormal durante la gestación se caracterizó por la toma aleatoria de biopsias cuya frecuencia de falsos negativos para lesiones invasoras del 8 al $40 \%$ era inaceptable (2).

Durante los años 60 dado que la colposcopia no se usaba mucho en algunos países, sobre todo en Norteamérica, la conducta ante un resultado de citología anormal solía implicar una conización diagnóstica. Las complicaciones fueron muchas y significativas tanto para la madre como para el feto: hemorragia, infección, estenosis e incompetencia cervical, corioamnionitis, flebitis y embolismo pulmonar, aborto, trabajo de parto prematuro y prematurez y mortinatos. Las complicaciones perinatales ocurrieron en el $19.4 \%$, y en total entre un 20 y $30 \%$ de las pacientes sufrieron algunas o varias de estas complicaciones (2). Además las posibilidades de enfermedad residual (por compromiso de los bordes) variaba entre un 50 y el $68 \%$ lo cual implicaba una nueva ronda terapéutica con reconización o histerectomia después de terminada la gestación.

Esto obligó a un esquema más conservador que incluyó a la colposcopia a partir de la segunda década de los años setenta de modo que solo las citologías con cambios de alto grado o cáncer eran remitidas para estudio y solo se tomaban biopsias si colposcópicamente se sospechaba la invasión. Las citologías durante la gestación con sospecha de lesiones de bajo grado no se estudiaban y se seguían con controles cada tres meses. DePetrillo y colaboradores en 1975 marcaron un hito e inauguraron esta tercera etapa al demostrar cómo la colposcopia era segura durante la gestación pues ningún carcinoma pasó desapercibido durante el examen de 329 casos. Redujo del 100\% al $3.2 \%$ la frecuencia de conización durante el embarazo la cual se hizo con indicación estrictamente diagnóstica (3). Hacker en 1982 confirmó estos hallazgos al evaluar todos los reportes publicados posteriormente; el análisis de varios trabajos con 1064 colposcopias hechas durante la gestación mostró una precisión diagnóstica para excluir cáncer del $99.8 \%$ porque solo dos carcinomas en estadio IA no fueron detectados durante el examen; solo se requirieron $3.9 \%$ cteconizaciones y las complicaciónes asociadas a la colposcopia sólo representó el 0.6 \% (2). McDonell en 1981 consideró que no era necesario estudiar con biopsia las citologías con anomalías Pap III y recomendó seguimiento citológico para estas pacientes (4) acentuando esta era conservadora reacción a una época de complicaciones.

Después de diez años de manejo conservador, Benedet en 1987 notó que no tomar biopsia de manera más liberal durante la colposcopia durante la gestación, sobre todo si el personal que lo hacía no tenía mucha experiencia, podía dejar pasar algunos carcinomas. Este autor reportó varios casos de cáncer cervical diagnosticados durante el puerperio en pacientes seguidas únicamente con colposcopia, sin biopsia, durante la gestación y no atribuibles a progresión desde una NIC, fenómeno raro durante el embarazo, sino a errores de apreciación del colposcopista (5). Además LaPollax en 1988 reportaron que $0.2 \%$ de las anormalidades menores en la citología durante la gestación mostraron carcinomas al ser estudiadas mediante colposcopia y biopsia sistemática (6).

Pasamos así a una nueva etapa de moderación en los años 90 donde toda anormalidad citológica debía ser evaluada e idealmente biopsiada con guía colposcópica. Esta cuarta era fue inaugurada por Ecónomos en 1993 con su experiencia de 612 gestantes, hasta ahora la mayor serie publicada, donde se confirmaron las sugerencias de anteriores autores. En este informe se biopsiaron el $73 \%$ de las embarazadas con citología anormal, $100 \%$ de las cuales fueron evaluadas por colposcopia; la concordancia entre la impresión colposcópica y el resultado de la biopsia fue del $95 \%$. Solo retornó a estudio durante el puerperio el $50 \%$ de las pacientes con diagnóstico previo de NIC III; 112 mujeres fueron sometidas a conización o histerectomía y en ninguna de ellas se encontró carcinomas no sospechados durante el período de gestación. Solo dos pacientes requirieron conización diagnóstica (7).

El cuello uterino de la mujer gestante presenta grandes cambios durante la evolución del embarazo. Ocurren notorias modificaciones histológicas y morfolpgicas que hacen del examen colposcópico un procedimiento difícil. Histológicamente el espesor del epitelio pavimentoso exocervical aumenta debido a la mayor actividad basal, donde son frecuentes las mitosis; en el endocervix, hay incremento en el número de las criptas glandulares y en el tamaño de las mismas, la superficie epitelial es hiperplásica, a veces simulando cambios adenomatosos.

Hay una notoria activación del proceso de metaplasia escamosa y el estroma neovascularizado es infiltrado por células inflamatorias y ocurre decidualización, al parecer de origen embrionario, entre un 10 y $34 \%$.

Morfológicamente el cuello uterino gestante es violáceo con placas blanquecinas a diferentes niveles, producto de la decidualización; aparecen áreas extensas de ectopia o se aumentan las previas, y sobre la misma, aparecen numerosos quistes de retención y formaciones polipoides de diferente tamaño y longitud. Aumenta el volumen de las papilas cilíndricas con la producción de abundante moco adherente; frecuentemente están sobreinfectadas y su fragilidad hace que sangren fácilmente. Hay producción activa demetaplasia escamosa inmadura con destrucción de elementos previamente formados. No hay imágenes específicas en las zonas de transformación anormal; las imágenes colposcópicas son complejas, mezclas de varios patrones y con bordes imprecisos. "La identificación colposcópica de las posibles áreas de neoplasia intra-epitelial y cáncer es difícil y es prácticamente imposible determinar, antes de la biopsia, el grado de lesión histológica. Todos los cambios a nivel del estroma, del epitelio 
glandular y del estratificado habrán desaparecido durante las siguientes 3 a 4 semanas posteriores al parto quedando únicamente en el cuello las secuelas traumáticas y las imágenes en regresión de las lesiones gravídicas" (8).

El propósito de la valoración colposcópica después de un frotis anormal durante la gestación, como dijimos antes, es excluir cáncer y por lo tanto es de importancia capital la exploración colposcópica durante el embarazo. La biopsia, cuando se realiza, deberá ser dirigida a las zonas de mayor preocupación, ya que no se ofrece tratamiento hasta el puerperio para las lesiones NIC, un retraso de 6 a 12 meses en el tratamiento definitivo de un carcinoma invasor temprano, puede tener consecuencias lamentables para una embarazada joven; es por tanto de suma importancia la exactitud y la confiabilidad del examen.

Debe hacerse valoración colposcópica en toda gestante con citología anormal. Si los cambios citológicos sugieren ASCUS o lesión de bajo grado, la necesidad de tomar o no biopsia dependerá de los hallazgos colposcópicos; si estos sugieren cambios mayores o invasión la biopsia necesariamente deberá hacerse. Cuando la citología sugiere lesión de alto grado siempre deberá tomarse biopsia dirigida.

La colposcopia durante la gestación exige de técnicas especiales para la total exposición y valoración del cuello uterino. Ya que rara vez el examen es insatisfactorio y por el riesgo inherente al procedimiento, el curetaje endocervical está contraindicado. Si la biopsia sugiere o confirma microinvasión, la conización diagnóstica se impone. Suele hacerse en el segundo trimestre y a pesar de los riesgos, la definición del estadio es prioritaria. Ocurre con frecuencia el compromiso de los bordes y hasta el momento actual la técnica más socorrida es la de bisturí frío.

Se ha reunido suficiente experiencia en el manejo de la citología anormal con colposcopia y biopsia durante la gestación en el centro de patología cervical de la E.S.E. Metrosalud en la unidad hospitalaria de Castilla y por lo tanto se diseñó una investigación descriptiva a fin de mostrar si es un método adecuado y seguro de evaluación y seguimiento, y si permite planear un manejo satisfactorio de la NIC evitando procedimientos que pudieran aumentar la morbilidad maternofetal. No hay información en nuestro medio, sobre la precisión de la colposcopia en el diagnóstico de la patología cervical durante la gestación.

\section{Materiales y métodos}

Se evaluaron las historias clínicas de las pacientes gestantes que asistieron a examen colposcópico por citología cervicouterina anormal durante el período de Mayo de 1991 a Junio de 1998 en el centro de patología cervical de Metrosalud; la información sobre cada una de las variables incluidas se consolidó en un formulario diseñado para efecto del estudio y los datos vaciados en una base creada en el programa informático Epi-info versión 5.

El objetivo del estudio fue examinar la exactitud de la colposcopia en la evaluación y seguimiento de la paciente embarazada con citología anormal, y para tal efecto se investigaron los factores de riesgo para lesión intraepitelial, edad a la que se presentan con mayor frecuencia las anomalías citológicas, cual fue la anormalidad más frecuente, la asociación entre los hallazgos citológicos y loscolposcópicos, el porcentaje de pacientes que requirieron biopsia, determinar el manejo según el resultado histólógico, la correlación entre los hallazgos colposcópicos durante el embarazo y el puerperio, y finalmente establecer la asociación entre los hallazgos citológicos y colposcópicos y el diagnóstico definitivo.

Para cada objetivo específico se hicieron cruces estadísticos entre variables y los resultados presentados en tablas con sus respectivos cálculos porcentuales.

Al final del estudio se consultó la bibliografía disponible sobre el tema y se discutieron los hallazgos comparándolos con los reportes de la literatura.

\section{Resultados}

Durante el período de estudio (20 de Mayo de 1991 a 30 de Junio de 1998), 1943 pacientes fueron remitidas al centro de patología cervical de la empresa social del estado Metrosalud en la unidad hospitalaria de Castilla para examen colposcópico por el resultado anormal en la citología, de las cuales 151 fueron mujeres embarazadas lo que representa una frecuencia del 7,8\%; de éstas se investigaron 127 pacientes y 24 fueron excluidas por datos incompletos en la historia clínica.

El rango de edad de estas mujeres en el momento de la consulta estaba entre los 14 y $\operatorname{los} 40$ años, con una edad promedio de 24 años. El mayor número de mujeres embarazadas con anormalidad citológica estaba en el grupo de edad de 16 a 25 años, 57,5\% (ver tabla No. 1).

El resultado citológico más frecuente fue el sugestivo de LIE de bajo grado (63\%), que predominó en el grupo de 16 a 25 años (55 pacientes); la citología sugestiva de LIE de alto grado se presentó en el 34,6\%, más frecuente en el grupo de 26 a 35 años (22 pacientes). Resaltamos como el LIE de alto grado se incrementa con la edad (ver tabla No. 1).

Tabla $0010 \mathrm{i01}$

GRUPO DE EDAD Y RESULTADO DE LA CITOLOGIA CERVICO UTERINA E. S. E. METROSALUD-CASTILLA. MEDELLIN 1991-1998

\begin{tabular}{|c|c|c|c|c|c|c|c|c|c|c|}
\hline \multirow{3}{*}{$\begin{array}{l}\text { GRUPO EDAD } \\
\text { EN AÑOS }\end{array}$} & \multicolumn{10}{|c|}{ RESULTADO DE LA CITOLOGIA } \\
\hline & \multicolumn{2}{|c|}{ ASCUS } & \multicolumn{2}{|c|}{ LEI BAJO ${ }^{\circ}$} & \multicolumn{2}{|c|}{ LEI ALTO ${ }^{\circ}$} & \multicolumn{2}{|c|}{ INVASOR } & \multicolumn{2}{|l|}{ TOTAL } \\
\hline & $\#$ & $\%$ & \# & $\%$ & \begin{tabular}{|l|l|}
$\#$ & -1 \\
\end{tabular} & $\%$ & \begin{tabular}{|l|l|}
$\#$ & -1 \\
\end{tabular} & $\%$ & \#| & $\%$ \\
\hline$<15$ & 0 & 0 & 3 & 100 & 0 & 0 & 0 & 0 & 3 & 2,4 \\
\hline $16-25$ & 0 & 0 & 55 & 75,3 & 18 & 24,7 & 0 & 0 & 73 & 37,5 \\
\hline $26-35$ & 1 & 2,3 & 20 & 45,4 & 22 & 50 & 1 & 2,3 & 44 & 34,6 \\
\hline$>35$ & 1 & 14,2 & 3 & 28,6 & 4 & 57,2 & 0 & 0 & 7 & 5,6 \\
\hline TOTAL & 2 & 1,6 & 80 & 63 & 44 & 34,6 & 1 & 0,8 & 127 & 100 \\
\hline
\end{tabular}

Se registraron los factores de riesgo para el resultado anormal de la citología en la paciente gestante. El promedio de edad para la primera relación sexual fue de 22 años, y el promedio de compañeros sexuales fue de 2. De las 127 pacientes, 27(21,2\%) tenían antecedente de tabaquismo. Se registró el dato de enfermedades de transmisión sexual sólo en 65 de las 127 pacientes, y de ellas resultaron positivas 20 pacientes $(30.7 \%)$.

Se encontró que el mayor número de pacientes habían tenido su primer coito antes de los 25 años (113 pacientes,89\%).El mayor número de pacientes $(53,5 \%)$ había tenido sólo un compañero sexual y sólo 5,8\% habían tenido 3 ó más compañeros. 
Se analizaron en forma conjunta los factores de riesgo: edad del primer coito, tabaquismo y número de compañeros sexuales; al realizar este cruce se encontró que de las 127 pacientes con anormalidad en la citología vaginal durante la gestación, 107 de ellas $(84,2 \%)$ tenían uno ó más factores de riesgo para lesiones intraepiteliales y sólo 16 no tenían ningún factor.

A todas las pacientes (127), se les hizo examen colposcópico, la mayor parte de las cuales (55 pacientes, $43,3 \%$ ) se realizaron entre las 13 y 26 semanas; se realizaron 44 colposcopias $(34,6 \%)$ en el tercer trimestre y 28 $(22,1 \%)$ en el primero.

La tabla No. 2 resume las impresiones colposcópicas para todas las embarazadas con citología anormal examinadas.

Todas las pacientes tuvieron una colposcopia satisfactoria. Sesenta y dos pacientes de 80 con citología de LIE

Tabla 0010102

CORRELACION ENTRE LA CITOLOGIA Y LA COLPOSCOPIA EN PACIENTES GESTANTES. E. S. E. METROSALUD-CASTILLA, MEDELLIN 1991-1998

\begin{tabular}{|c|c|c|c|c|c|c|c|c|c|c|}
\hline \multirow{3}{*}{ CITOLOGIA } & \multicolumn{10}{|c|}{ IMPRESIÓN COLPOSCOPICA } \\
\hline & \multicolumn{2}{|c|}{ NEGATIVA } & \multicolumn{2}{|c|}{$\begin{array}{l}\text { CAMBIOS } \\
\text { MENORES }\end{array}$} & \multicolumn{2}{|c|}{$\begin{array}{l}\text { CAMBIOS } \\
\text { MAYORES }\end{array}$} & \multicolumn{2}{|c|}{ INVASION } & \multicolumn{2}{|c|}{ TOTAL } \\
\hline & $\#$ & $\%$ & \# & $\%$ & \begin{tabular}{|l|l|}
$\#$ & \\
\end{tabular} & $\%$ & \# & $\%$ & $\#$ & $\%$ \\
\hline ASCUS & 0 & 0 & 2 & 100 & 0 & 0 & 0 & 0 & 2 & 1,6 \\
\hline LIE BAJO ${ }^{\circ}$ & 3 & 3,7 & 62 & 77.5 & 14 & 17,5 & 1 & 1.3 & 80 & 66.3 \\
\hline LIE ALTO० ${ }^{\circ}$ & 0 & 0 & 16 & 36,3 & 25 & 56,8 & 3 & 6.9 & 44 & 346 \\
\hline INVASION & 0 & 0 & 0 & 0 & 1 & 100 & 0 & 0 & 1 & 0,7 \\
\hline TOTAL & 3 & 2,4 & 80 & 63 & 40 & 31,5 & 4 & $\overline{3,1}$ & 127 & 100 \\
\hline
\end{tabular}

de bajo grado tuvieron una colposcopia con cambios menores $(77,5 \%)$ y una paciente fue sospechosa de invasión; 28 pacientes de 42 con citología de alto grado tuvieron colposcopia que mostraron cambios mayores o invasión $(63,6 \%)$. Sólo una paciente tenía citología sugestiva de carcinoma y la colposcopia mostró cambios mayores y no invasión. Aunque la mayoría de las pacientes con citología de bajo grado mostraron colposcopias con cambios menores y la mayoría de las pacientes con citología de alto grado mostraron cambios mayores en la colposcopia, no hay coincidencia entre el resultado de la citología y la impresión colposcópica (ver tabla No. 2).

De las 127 pacientes se tomaron biopsia en 102 de ellas $(80,3 \%)$. De las 83 pacientes con cambios menores, se tomó biopsia a 59 de ellas $(71,1 \%)$; de las 44 pacientes con cambios mayores o invasión se tomó biopsia a 43 de ellas $(97,7 \%)$ (ver tabla No. 3).

\section{Tabla $0010 \mathrm{i} 03$}

TOMA DE BIOPSIA SEGUN LOS HALLAZGOS COLPOSCOPICOS EN PACIENTES GESTANTES. E. S. E. METROSALUD-CASTILLA, MEDELLIN 1991-1998

\begin{tabular}{|c|c|c|c|c|c|c|}
\hline \multirow{3}{*}{ COLPOSCOPIA } & \multicolumn{6}{|c|}{ BIOPSIA } \\
\hline & \multicolumn{2}{|l|}{ NO } & \multicolumn{2}{|c|}{ SI } & \multicolumn{2}{|l|}{ TOTAL } \\
\hline & $\#$ & $\%$ & $\#$ & $\%$ & \begin{tabular}{|l|l|}
$\#$ & \\
\end{tabular} & $\%$ \\
\hline $\begin{array}{l}\text { NEGATIVA O CAMBIOS } \\
\text { MENORES }\end{array}$ & 24 & 29,9 & 59 & 71,1 & 83 & 65,3 \\
\hline $\begin{array}{l}\text { CAMBIOS MAYORESO } \\
\text { INVASION }\end{array}$ & 1 & 2,3 & 43 & 97,7 & 44 & 34,7 \\
\hline TOTAL & 25 & 19,7 & 102 & 80,3 & 127 & 100 \\
\hline
\end{tabular}

Se obtuvo resultado histológico en 99 de las 102 pacientes sometidas a biopsia, 3 no trajeron el informe. Los diagnósticos fueron: infección por papiloma virus humano (PVH) 33 (33\%), NIC I 22 (22\%), NIC II 13 (13\%), NIC III 14 (14\%), microinvasión $5(5 \%)$ e invasión $2(2 \%)$ y 10 biopsias fueron

$$
\text { Tabla 0010i04 }
$$

\section{CORRELACION ENTRE LOS HALLAZGOS COLPOSCOPICOS Y EL RESULTADO DE LA BIOPSIA. E. S. E. METROSALUD-CASTILLA, MEDELLIN 1991-1998}

\begin{tabular}{|c|c|c|c|c|c|}
\hline \multirow{2}{*}{\multicolumn{2}{|c|}{ BIOPSIA }} & \multirow{2}{*}{\multicolumn{4}{|c|}{ COLPOSCOPIA }} \\
\hline & & & & & \\
\hline & & menores & mayores & Invasor & Total \\
\hline sin dato & & 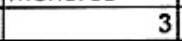 & (2) & 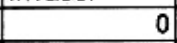 & 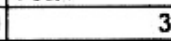 \\
\hline \multirow[t]{2}{*}{ Normal } & $\#$ & 9 & 1 & 0 & 10 \\
\hline & $\%$ & 16 & 2,6 & 0 & 10 \\
\hline \multirow[t]{2}{*}{$\mathrm{PVH}$} & \# & 26 & 7 & 0 & 33 \\
\hline & $\%$ & 46,5 & 18 & 0 & 33 \\
\hline \multirow[t]{2}{*}{$\mathrm{N}|\mathrm{C}|$} & \# & 15 & 7 & 0 & 22 \\
\hline & $\%$ & 26,8 & 18 & 0 & 22 \\
\hline \multirow[t]{2}{*}{ NIC II } & $\#$ & 5 & 8 & 0 & 13 \\
\hline & $\%$ & 8,9 & 20,5 & 0 & 13 \\
\hline \multirow[t]{2}{*}{ NIC III } & $\#$ & 1 & 13 & 인 & 14 \\
\hline & $\%$ & 1,8 & 33,4 & 0 & 14 \\
\hline \multirow[t]{2}{*}{ Microinvasor } & $\#$ & 0 & 3 & 2 & 5 \\
\hline & $\%$ & 0 & 7,7 & 50 & 5 \\
\hline \multirow[t]{2}{*}{ Invasor } & $\#$ & 0 & of & 2 & 2 \\
\hline & $\%$ & 0 & 0 & 50 & 2 \\
\hline \multirow[t]{2}{*}{ total } & $\#$ & 56 & 39 & 4 & 99 \\
\hline & $\%$ & 56,5 & 39,3 & 4,2 & 100 \\
\hline
\end{tabular}

La tabla No. 4 ilustra la asociación entre los hallazgos colposcópicos y el diagnóstico histológico en la biopsia dirigida durante el embarazo. Se obtuvieron 47 biopsias anormales en colposcopias que mostraron cambios menores, detectándose 6 casos $(10,7 \%)$ de lesiones NIC II-III y ningún carcinoma (0\%) y 42 biopsias anormales en colposcopias que reportaron cambios mayores o invasión y se detectaron 21 casos $(46.7 \%)$ de lesiones NIC II-III y 7 invasores $(18,5 \%)$ (ver tabla No. 5).

\section{Tabla $0010 \mathrm{i} 05$ \\ CORRELACION}

CITOLOGIA-COLPOSCOPICA-BOPSIA EN GESTANTES CON CITOLOGIA ANORMAL. E. S. E. METROSALUD-CASTILLA, MEDELLIN 1991-1998

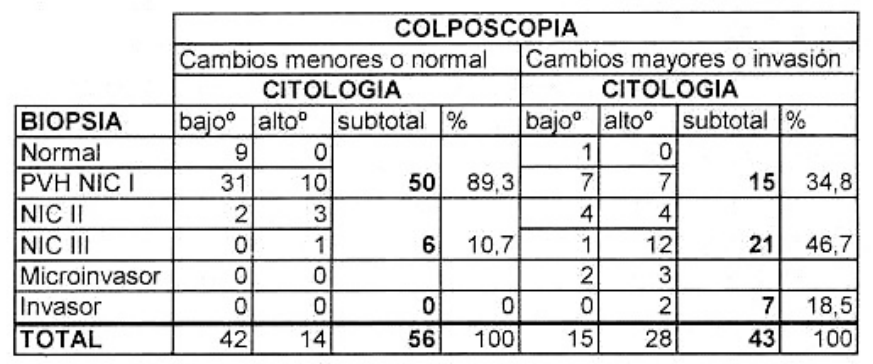

Nota: bajo incluye ASCUS alto $^{\circ}$ incluye sospecha de invasión

Se observó que la probabilidad de encontrar un carcinoma es independiente del resultado de la citología: 2 en 
citología de bajo grado y 5 en citología de alto grado. En cambio, la impresión colposcópica predijo la probabilidad de encontrar carcinomas, y no se presentaron cuando la colposcopia mostró cambios menores o normalidad (Ver tabla No. 5).

Se hizo un análisis separado de las pacientes estudiadas por citología con cambios de ASCUS y LIE de bajo grado para determinar las posibilidades de lesiones mayores en estas pacientes. En este grupo predominó el criterio colposcópico y no todas fueron biopsiadas; de las 83 pacientes con cambios citológicos de ASCUS o lesión de bajo grado se tomó biopsia a 59 pacientes $(71.1 \%)$ de las cuales 3 no fueron informadas, 9 fueron negativas y 47 anormales: 7 con NIC II-III $(12,3 \%)$ y 2 con cáncer $(3,5 \%)$ datos que resaltan la importancia de incluir estos resultados citológicos en el protocolo de colposcopia.

La colposcopia (dividida en tres grados: normal o menores, mayores e invasión) correlacionó, sin ningún grado de variación, con la biopsia dirigida (dividida también en tres grados: normal-PVH- NIC I, NIC II-III e invasión) en el $75.7 \%$ de los casos.

Para efectos de comparación con otros estudios sobre el valor diagnóstico de la colposcopia en pacientes gestantes se estableció una tabla donde se determinó el grado de concordancia de la impresión colposcópica con la biopsia considerada está como criterio de enfermedad. La prueba (en este caso la colposcopia) era positiva si se observaban cambios mayores o invasión y la enfermedad estaba presente si en la biopsia dirigida se encontraba NIC II-III o invasión (ver tabla No 6). Del análisis de ella se calcularon en este estudio una sensibilidad del $82.4 \%$ y una especificidad del $76.9 \%$ con un valor predictivo negativo del $89.3 \%$ y uno positivo del $65.1 \%$. La prevalencia de enfermedad importante en la población de alto riesgo estudiada fue del $34.3 \%$.

Cinco pacientes con diagnóstico histológico,de cáncer microinvasor en la biopsia dirigida fueron planeadas para conización diagnóstica y se realizó el procedimiento con técnica fría tradicional a 3 de ellas, con radiofrecuencia a una y otra no aceptó el procedimiento diagnóstico. Los resultados histológicos de estos procedimientos fueron: un carcinoma microinvasor con invasión < de $3 \mathrm{~mm}$ y borde positivo para NIC III; esta paciente se observó durante el embarazo y se le realizó histerectomía en el puerperio. Otra paciente, con carcinoma microinvasor e invasión < de $2 \mathrm{~mm}$ y vértice positivo para NIC III no volvió a seguimiento luego del cono. En otra, el cono reportó NIC III con borde positivo para NIC III y tampoco retornó a seguimiento. La última paciente reportó microinvasión < de $3 \mathrm{~mm}$ con bordes negativos y se dejó como tratamiento definitivo.

Las pacientes con diagnóstico histológico de cáncer fueron biopsiadas a las semanas 20 y 29 y ambas fueron remitidas a un hospital de III nivel; una fue sometida a histerectomía radical postcesárea a la semana 34 y la otra a radioterapia luego de cesárea corporal a la misma semana de gestación.

El manejo durante el embarazo según el resultado de la biopsia fue: ácido tricloroacético 49 pacientes $(38,6 \%)$, observación 39 pacientes $(39,7 \%)$ incluyendo en ellas los 5 microinvasores; 10 pacientes no necesitaron manejo porque sus biopsias fueron negativas y 3 no volvieron al control.

Tabla 0010i06

CONCORDANCIA DIAGNOSTICA DE LA COLPOSCOPIA EN COMPARACION CON LA BIOPSIA DIRIGIDA DURANTE LA GESTACION. E. S. E. METROSALUD-CASTILLA, MEDELLIN 1991-1998

\begin{tabular}{|c|c|c|c|}
\hline \multirow[b]{2}{*}{ Prueba (Colposcopia) } & \multicolumn{3}{|c|}{ Enfermedad (Biopsia) } \\
\hline & $\begin{array}{c}\text { Positivo } \\
\text { Nic II-III/ invasión }\end{array}$ & $\begin{array}{c}\text { Negativo } \\
\text { Normal,PVH,Nic I } \\
\end{array}$ & total \\
\hline $\begin{array}{c}\text { Positivo } \\
\text { Cambios mayores/invasión }\end{array}$ & 28 (a) & 15 (b) & 43 \\
\hline $\begin{array}{c}\text { Negativo } \\
\text { Cambios menores/normal }\end{array}$ & 6 (c) & 50 (d) & 56 \\
\hline total & 34 & 65 & 99 \\
\hline
\end{tabular}

Sensibilidad: a/a + c x 100, tasa de detección.

Especificidad: $d / b+d \times 100$, tasa de exclusión.

V.P.N.: $\mathrm{c} / \mathrm{c}+\mathrm{d} \times 100$, Posibilidad de no lesión ante una prueba negativa.

V.P.P.: $\quad a / a+b \times 100$, Posibilidad de lesión ante la prueba positiva.

Dos pacientes con cáncer fueron sometidas a histerectomía radical y radioterapia, como se mencionó anteriormente (ver tabla No. 6).

De las 39 pacientes a quienes se aplazó el tratamiento sólo regresaron 24 (61,5\%); se les hizo colposcopia en el puerperio todas las pacientes (100\%) y la misma no fue mayor que la hecha durante el embarazo en ningún caso: los resultados fueron siempre iguales en 23 pacientes y menores en 1 caso.

De las 39 pacientes a las que se les aplazó el tratamiento hasta el puerperio se trataron definitivamente 24 pacientes, la mayoría con manejo quirúrgico (17 pacientes, $70.8 \%$ ). La mayor deserción se observó entre las pacientes con diagnóstico histológico de NIC II, ya que sólo regresaron 3 de 13 pacientes a control (Ver tabla No. 7).

Hay diagnóstico histológico final en 16 de las 17 intervenidas quirúrgicamente (1 no trajo resultado histológico) y en ellas sólo se registró lesión mayor que la diagnosticada en el embarazo en dos pacientes con diagnóstico histológico previo de NIC II cuyo informe histológico definitivo registró NIC III, pero en ningún caso carcinoma.

\section{Discusión}

El aplazamiento de la procreación a edades más tardías entre las mujeres de la sociedad actual, la mayor frecuencia de cáncer cervical a edades más jóvenes y el cambio en la moda de edad de presentación de las lesiones epiteliales de alto grado, está determinando una mayor necesidad de estudiar colposcópicamente a las mujeres en gestación. La colposcopia durante el embarazo supone un reto para el médico especialista por los cambios fisiológicos que hacen más difícil la técnica y la interpretación del examen así como la decisión de tomar o no biopsias dirigidas.

El número de remisiones de gestantes para estudio colposcópico al centro de patología cervical de la ESE 


\section{Tabla $0010 \mathrm{i} 07$ \\ DIAGNOSTICO HISTOLOGICO Y MANEJO DURANTE EL EMBARAZO EN PACIENTES CON CITOLOGIA ANORMAL DURANTE LA GESTACION. E. S. E. METROSALUD-CASTILLA, MEDELLIN 1991-1998}

\begin{tabular}{|c|c|c|c|c|c|c|c|}
\hline \multirow[b]{2}{*}{ BIOPSIA } & \multicolumn{7}{|c|}{ TRATAMIENTO } \\
\hline & $\begin{array}{l}\frac{0}{0} \\
\frac{\pi}{0} \\
\frac{5}{6}\end{array}$ & 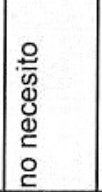 & $\frac{5}{4}$ & 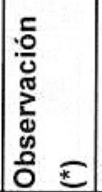 & 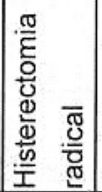 & $\begin{array}{l}\frac{\pi}{0} \\
\frac{0}{\pi} \\
\frac{\pi}{0} \\
\frac{0}{0} \\
\frac{\pi}{10} \\
\alpha\end{array}$ & 要 \\
\hline No necesito & & 17 & 8 & & & & 25 \\
\hline Sin dato & 3 & & & & & & 3 \\
\hline Normal & & 10 & & & & & 10 \\
\hline \begin{tabular}{|l}
$\mathrm{PVH}$ \\
\end{tabular} & & 7 & 26 & & & & 33 \\
\hline NIC I & & & 15 & 7 & & & 22 \\
\hline NIC II & & & & 13 & & & 13 \\
\hline NIC III & & & & 14 & & & 14 \\
\hline Microinvasor & & & & 5 & & & 5 \\
\hline Invasor & & & & & 1 & 1 & 2 \\
\hline Total & 3 & 34 & 49 & 39 & 1 & 1 & 127 \\
\hline
\end{tabular}

(*) tratamiento diferido hasta después del parto

Metrosalud - Castilla $(7,8 \%)$ es muy similar a la reportada por otros centros (7). De igual manera se destaca como la mayoría de gestantes es muy joven, 59,9 \% eran menores de 25 años. Un número importante de pacientes consultaron tardíamente $(34,6 \%$ en el tercer trimestre), lo cual es importante ya que después de la semana 29 el examen colposcópico, por lo avanzado de la gestación, es más difícil. Esto indica una remisión tardía y que no se está sugiriendo la citología a las gestantes al iniciar el control prenatal. En otros reportes sólo el $9 \%$ de las colposcopias fueron realizadas en el tercer trimestre (7).

Como en otros estudios, la edad temprana del primer coito (antes de los 20 años) aparece como factor de riesgo dominante de citología anormal entre las gestantes. En el reporte de Bertini-Oliveira (9) $65 \%$ de las pacientes ya eran sexualmente activas antes de los 19 años; en este reporte $81,9 \%$ de las gestantes ya lo eran.

A diferencia de las no gestantes donde siempre debe tomarse biopsias dirigidas durante el examen colposcópico, en la gestación esta decisión es discrecional aunque los riesgos de hemorragia importante y trabajo de parto son mínimos, no se justifican si los hallazgos colposcópicos son menores.

En este estudio 80,3\% de las pacientes fueron biopsiadas. En otros reportes la cifra fue muy similar: $73 \%$ (7).

En vista que la probabilidad de cáncer entre gestantes con cambios colposcópicos menores fue $0 \%$ en el futuro el número de biopsias en este grupo debería disminuirse significativamente (actualmente es de 71,1\%).

A diferencia de otros recientes investigadores (10), en este medio no es loable seguir sólo con citología las gestantes con ASCUS o sospecha de lesión de bajo grado. En este reporte la mayoría de las gestantes $(64,6 \%)$ consultaron por este hallazgo y en 2 de ellas se encontró carcinoma microinvasor (3.5\%). Si bien supone un costo elevado, estos hallazgos lo justifican. En el reporte de Jain (10) 253 gestantes con citología de bajo grado fueron investigadas con colposcopia, ninguna de las 67 biopsias tomadas reportó carcinoma. En el estudio de Ecónomos (7) un carcinoma fue diagnosticado en una gestante con citología sugestiva de ASCUS.

El porcentaje de gestantes con cáncer en este estudio $(5,51$ $\%)$ es preocupante. En el reporte de Ecónomos (7) sólo 0.33\% de las gestantes con citología anormal tenían cáncer (2 de 617) y en el de Bertini-Oliveira (9) hecho en Latinoamérica, la frecuencia fue de 2,4\% (3 de 125). Esto implica hacer énfasis en las autoridades de salud de la importancia de la citología vaginal durante el control prenatal. Estos datos indican el curso epidemiológico que está tomando el cáncer cervical en nuestro medio.

La experiencia del centro con pacientes gestantes confirma la eficacia y la seguridad de la colposcopia durante la gestación. El grado de correlación colpohistológica fue aceptable, 4 de las 7 pacientes con carcinomas tuvieron una colposcopia sugestiva de invasión y las 3 restantes se asociaron a cambios mayores y fueron correctamente biopsiadas.

En este estudio se pudo medir la efectividad de la colposcopia para distinguir el tejido normal, con papilomavirus o NIC I de las lesiones de alto grado y carcinoma.

El examen colposcópico se hace sobre una población de alto riesgo que ha sido previamente seleccionada por la citología. Como prueba diagnóstica, su papel más importante es discriminar las lesiones notables que bien tratadas permiten prevenir el cáncer cervical de las que apenas se están iniciando y no son susceptibles de manejo conservador (estadíos IA2 o lB).

Fue así como la sensibilidad y la especificidad como también los valores predictivos negativo y positivo de la colposcopia durante el embarazo para reconocer lesiones mayores o cáncer son semejantes a lo informado en la literatura. Un reciente meta-análisis de ocho estudios reportó una sensibilidad del $79 \%$ y una especificidad del $67 \%$ un valor predictivo negativo de $85 \%$ y positivo del $57 \%$ (11), cifras muy cercanas a las reportadas en este informe.

Como el fin de la colposcopia durante la gestación es excluir la posibilidad de cáncer, en este reporte ese objetivo se cumplió aparentemente en el $100 \%$ de los casos. Ningún carcinoma no diagnosticado apareció entre las pacientes tratadas con cirugía en la cual removimos totalmente la zona de transformación durante el post-puerperio (16 pacientes) ni entre las que se controlaron colposcópicamente (ver tabla No. 8).

Puede resaltarse entonces la importancia de tomar siempre biopsia cuando la imagen colposcópica sugiera cambios mayores o invasión. Otros autores (3) propusieron el seguimiento sólo colposcópico; siguiendo esta recomendación Benedet falló en diagnosticar correctamente 5 carcinomas que fueron detectados en el postparto y aunque se postuló que las lesiones pudieron aparecer como evolución desde lesiones NIC III, esto es poco probable. Desde entonces se recomienda que la colposcopia sola, sin biopsia dirigida cuando está indicada, supone un riesgo importante de subestimar la severidad de las lesiones 
Tabla 0010i08

TRATAMIENTO DEFINITIVO Y DIAGNOSTICO HISTOLOGICO FINAL EN LAS PACIENTES SOMETIDAS A CIRUGIA, QUE CONSULTARON EN EMBARAZO CON CITOLOGIA ANORMAL. E. S. E. METROSALUD-CASTILLA, MEDELLIN 1991-1998

\begin{tabular}{|c|c|c|c|c|c|}
\hline TRATAMIENTO & No. & \multicolumn{2}{|c|}{ Diagnóstico biopsia } & \multicolumn{2}{|c|}{ Diagnostico final } \\
\hline Crioterapia & 2 & NIC I & 2 & & \\
\hline Radiofrecuencia & 3 & $\begin{array}{lll}\text { NIC II } \\
\text { NIC III }\end{array}$ & $\begin{array}{l}2 \\
1\end{array}$ & NIC III & 3 \\
\hline Cono frio & 4 & $\begin{array}{l}\text { NIC II } \\
\text { NIC III } \\
\text { Microinvasor }\end{array}$ & $\begin{array}{l}1 \\
2 \\
1\end{array}$ & $\begin{array}{l}\text { NIC II } \\
\text { NIC III } \\
\text { Microinvasor }\end{array}$ & $\begin{array}{l}1 \\
2 \\
1\end{array}$ \\
\hline Histerectomia & 10 & $\begin{array}{l}\text { NIC III } \\
\text { Microinvasor }\end{array}$ & $\begin{array}{l}9 \\
1\end{array}$ & $\begin{array}{l}\text { NIC III } \\
\text { Microinvasor }\end{array}$ & $\begin{array}{l}8 \\
1\end{array}$ \\
\hline Observación & 5 & $\mathrm{NICl}$ & 5 & & \\
\hline Total & 24 & & 24 & & 16 \\
\hline
\end{tabular}

Así cuando se tengan dudas, o la colposcopia sugiera cambios mayores o invasión, la biopsia debe ser tomada.

Destacamos la experiencia en este estudio con la conización diagnóstica durante el segundo trimestre (4 casos) en las que no se presentó morbilidad para la gestante ni su producto y la posibilidad de probar 2 técnicas diferentes (frío y radiofrecuencia). Se resalta la alta posibilidad de bordes comprometidos (75\%), seguramente ocasionado por lo limitado de la resección y el temor en dañar la evolución de la gestación. Pero no hay dudas, debe hacerse ante el reporte de microinvasión o sospecha de la misma para un correcto enfoque el resto del embarazo.

Finalmente, es importante que cada centro tenga un protocolo de evaluación y manejo de la citología anormal durante la gestación y personal entrenado y experimentado en la realización del examen en circunstancias tan particulares.

\section{Conclusiones}

5.1. La mayoría de pacientes embarazadas (57,5\%) que requirieron colposcopia en la gestación tienen entre 16 y 25 años y consultan con mayor frecuencia en el segundo trimestre de la gestación $(43,3 \%)$.

5.2. La mayoría de pacientes embarazadas $(64,6 \%)$ que requirieron colposcopia consultaron con resultado de lesión intraepitelial de bajo grado en su citología.

5.3. De los factores de riesgo conocidos para neoplasia intraepitelial cervical evaluados en este estudio el más frecuente fue el inicio de la actividad sexual antes de los 25 años; la mayoría de pacientes $(84,2 \%)$ tenían uno o más de los factores evaluados.

5.4. Se presentaron dos casos de cáncer entre las embarazadas con resultado de lesión de bajo grado en la citología.

5.5. La mayoría de las biopsias (89,2\%) tomadas durante la colposcopia en gestantes con cambios menores reportaron normalidad, PVH o NIC I, lo cual podría justificar el no tomar biopsia en estos casos.
5.6. Siempre debe tomarse biopsia cuando la colposcopia sugiere cambios mayores o invasión, independiente del resultado de la citología; de esta manera podrán diagnosticarse adecuadamente todos los carcinomas (7 en este estudio).

5.7. Los casos de sospecha de microinvasión en la biopsia, deben someterse a conización diagnóstica y la probabilidad de bordes positivos es alta (75\%).

5.8. Cuando se diagnostica NIC II o NIC III en la gestación, es seguro para las pacientes hacer control colposcópico y diferir el tratamiento para el puerperio ya que la probabilidad de progresión de la lesión a cáncer es casi nula.

5.9. Como protocolo de manejo en pacientes embarazadas con citología anormal proponemos:

5.9.1. Colposcopiaa todagestante con citología anormal.

5.9.2. La decisión de tomar o no biopsia dependerá de los hallazgos colposcópicos, independiente de lo reportado en la citología. Si la colposcopia muestra cambios menores sugerimos no tomar biopsia; si la colposcopia muestra cambios mayores o invasión tomar biopsia siempre.

5.10. El tratamiento dependerá del resultado de patología:

5.10.1. Si la biopsia informa PVH, NIC I, NIC II, NIC III o la colposcopia mostró cambios menores y no se tomó biopsia, se puede hacer seguimiento colposcópico y diferir el tratamiento hasta después del parto.

5.10.2. Si la biopsia informa cáncer, manejar el caso según los protocolos establecidos: si la biopsia

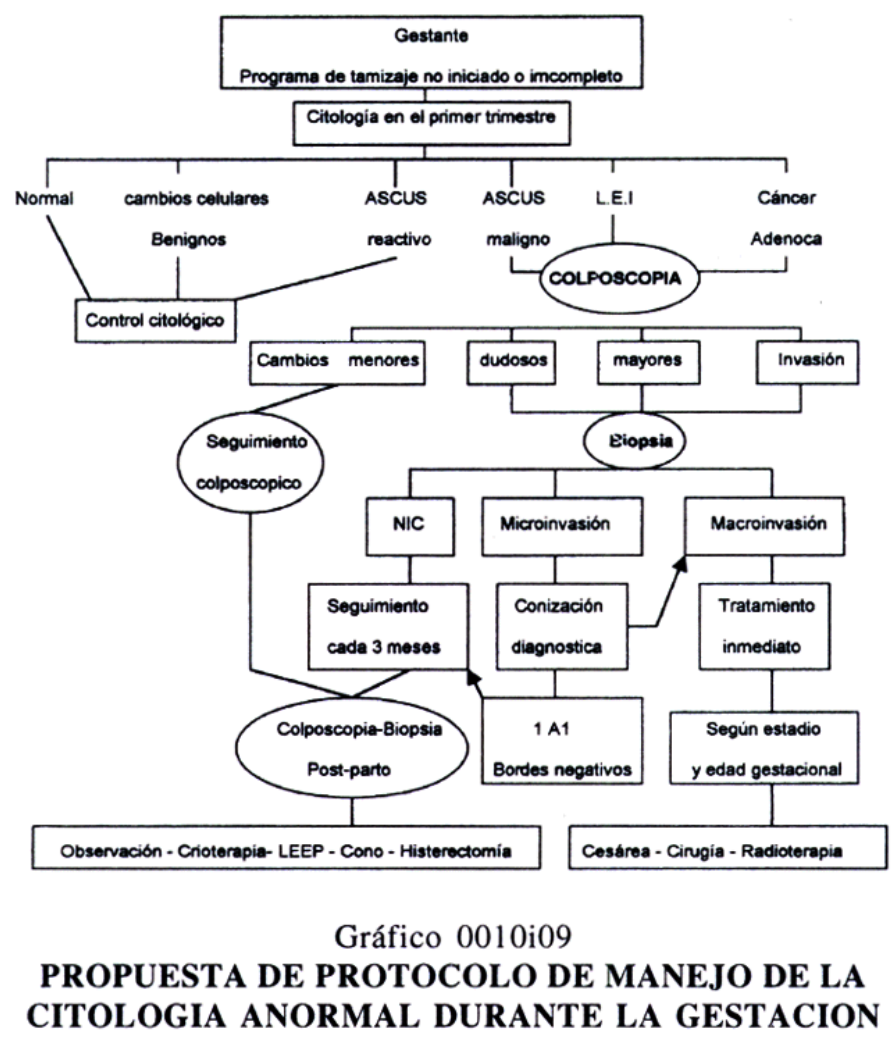

Centro de patología cervical y colposcopia, Metrosalud - Medellín, 2000. 
informa microinvasión, realizar conización diagnóstica (frío o radiofrecuencia según las circunstancias) para descartar macroinvasión; descartada ésta se hará seguimiento colposcópico y se difiere el tratamiento para después del parto; si la biopsia o el cono informan macroinvasión se manejará según la edad gestacional y el estadio como está establecido en los protocolos del Instituto Nacional de Cancerología (Ver el gráfico No 9).

\section{Recomendaciones}

6.1. Instruir a la comunidad y al personal médico y paramédico de la probabilidad de cáncer cervical durante la gestación después de los 25 años.
6.2. Recomendar la toma de citología en el primer control prenatal en el primer trimestre del embarazo en toda mujer cuyo programa de tamizaje esté incompleto o no se haya iniciado.

6.3. Remitir a colposcopia a toda embarazada cuyo resultado de citología sea anormal (ASCUS, ASGUS, lesión sugestiva de NIC o cáncer).

6.4. Instruir a la comunidad y al personal médico y paramédico que la toma de biopsia durante la gestación es segura.

6.5. Explicar que el manejo de la NIC en el embarazo, confirmada por colposcopia, se posterga para el postparto, pues el riesgo de progresión es casi nulo.

\section{BIBLIOGRAFIA}

1. Wright, C. et al. Colposcopia actual. Clínicas de ginecología y obstetricia. Interamericana, McGraw-Hill. México, 1993; 145-154.

2. Hacker, N. et al. Carcinoma of cervix asspciated with pregnancy. Obstet. and Gynecol. 1982; 59(6): 735-746.

3. DePetrillo, A. et al. Colposcopy evaluation of abnormal Papanicolaou test in pregnancy. Am. J. Obstet and Gynecol. 1975; 121(4): 441-445.

4. McDonnell, J.M. et al. Colposcopy in pregnancy: a twelve year review. Br. J. Obstet. Gynecol. Vol. 88. Pág. 414-420.

5. Benedet J.L, Selke P.A, Nickerson K.G. Colposcopic evaluation of abnormal Papanicolau smears in pregnancy. Am. J. Obstet. Gynecol. 1987; 157: 932-937.

6. LaPollax J.P. et al. Colposcopic managernent of anormal cervical cytology in pregnancy. J. Reprod. Med. 1988; 33: 301-306.
7. Economos, K. et al. Abnormal cervical cytology in pregnancy: A 17 year experiencie. Obstet. Gynecol. 1993; 81(6): 915-918.

8. Dexeus, S. et al. Tratado y atlas de patología cervical. Salvar editores. Barcelona, 1989; 293-297.

9. Bertini-Oliveira A. et al. Comparative evaluation of abnormal cytology, colposcopy and histopathology in preclinical cervical malignancy during pregnancy. Acta Cytol. 1982; 26(5): 636-644.

10. Jain A.G., Higgins R.Y., Boyle M.J. Management of low-grade squamous intraepithelial lesions during pregnancy. Am. J. Obstet. Gynecol. 1997; 177(2): 298-302.

11. Follen M., et al. Colposcopy for the diagnosis of squamous intraepithelial lesions: A meta-analysis. Obstet. and Gynecol. 1998; 91(4): 626-631. 